\title{
Advances in understanding the morphology of glomerular disease
}

\author{
DR TURNER*
}

From the Department of Pathology, Guy's Hospital Medical School, London Bridge, London SEI 9RT

SILVER IMPREGNATION TECHNIQUES

Before the introduction of renal biopsy our understanding of the morphology of glomerular disease was extremely limited. This could be blamed in part on the problems of interpretation of necropsy material obscured to a greater or lesser extent by post-mortem artefact. The introduction of renal biopsy made fresh renal tissue available and acted as a stimulus for the development of a variety of new histological techniques which have subsequently proved to be of great value. In 1957 Jones published his silver-impregnation method for the demonstration of glomerular basement membrane material ${ }^{1}$

*Present address: Department of Pathology, Musgrove Park Hospital, Taunton, Somerset. and illustrated many of the morphological changes which could occur in glomerular disease. I regard his technique as the most valuable single method in the day-to-day diagnosis of glomerular disease, provided it is used with a haematoxylin and eosin counterstain. Many people virtually waste the technique by using a weak light green counterstain which is almost useless. In the normal glomerulus, the silver impregnation technique clearly identifies the glomerular capillary wall and mesangial matrix while haematoxylin stains the various nuclei, and eosin stains the proteins.

In membranous glomerulonephritis, the Jones technique can be used to illustrate the argyrophilic "spikes" (Fig. 1) which classically project from the

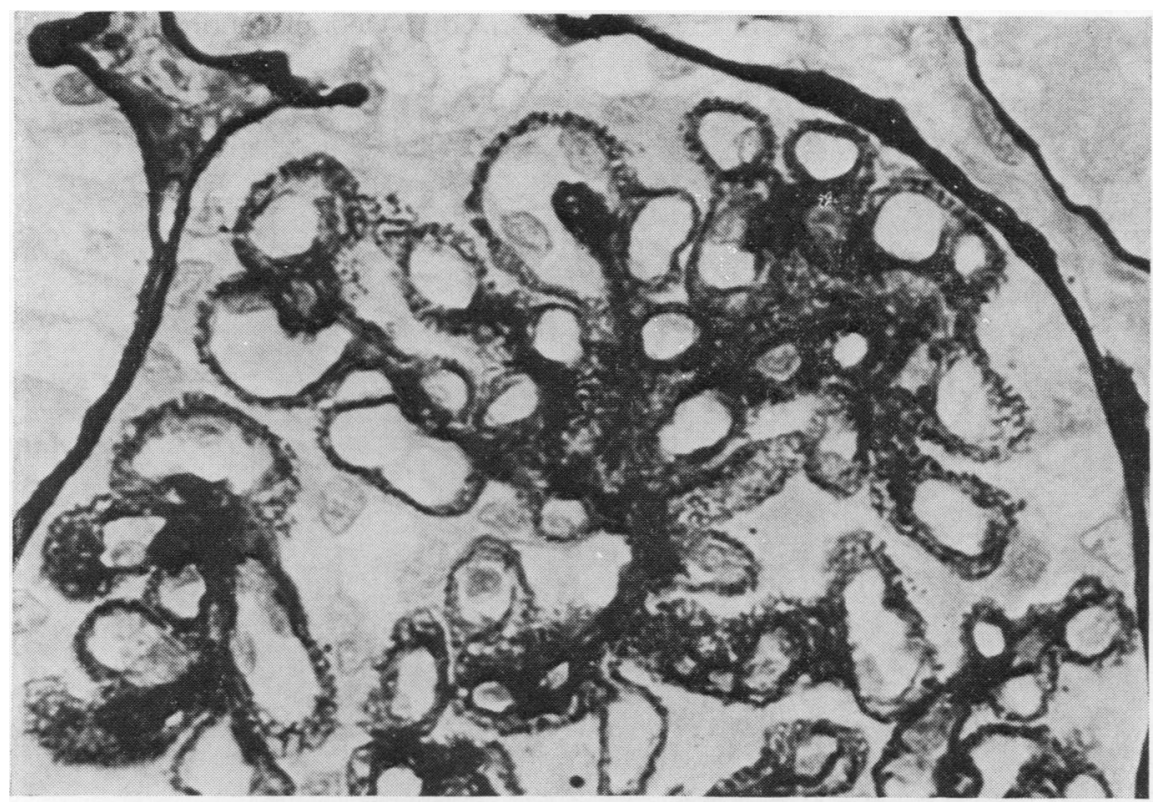

Fig. 1 Part of a glomerulus showing a complicated pattern of argyrophilic projections from the outer aspect of the capillary basement membranes in membranous glomerulonephritis. Jones' methenamine silver with haematoxylin and eosin counterstain $\times 560$. 
outer aspect of the glomerular basement membrane, but often the spikes are too small to be readily seen by light microscopy, or too complex, being woven into a chain-link pattern which is difficult to interpret.

It is important to appreciate that the Jones technique, like other silver impregnation techniques, is difficult to reproduce consistently. The longer the incubation is continued the more silver is deposited, and the basement membranes can look abnormally thick due to excessive deposition of silver. Therefore a reliable estimation of basement membrane thickness cannot be made by the Jones technique.

The advantage of using a haematoxylin and eosin counterstain is that the contrast between the red protein deposits in the glomerulus and the black argyrophilic basement membrane material enables them to be delineated. Thus in mesangiocapillary glomerulonephritis it is possible sometimes to identify the larger subendothelial deposits on the inner aspect of the glomerular capillary basement membranes. When the deposits are less evident it may still be possible to identify the double-contour effect produced by the mesangial cells laying down an additional layer of argyrophilic material in the subendothelial position, and also the lobular pattern produced by the increase in mesangial matrix and cells.

In the recurrent haematuria syndrome there may be very little to be seen in the glomeruli by light microscopy. However in some cases it is possible to identify the paramesangial deposits of IgA which bulge out from the side of the mesangium, and can be stained with eosin. Similarly in focal segmental glomerulosclerosis the contrast between the eosinophilic staining of the hyalinosis lesion and the surrounding sclerosis stained with silver, is of value in identifying this condition with a poor prognosis (Fig. 2).

The counterstaining principle is particularly helpful in the interpretation of linear dense deposit disease in which the abnormal material which is laid down in the glomerular capillary basement membrane fails to take up the silver stain and is stained therefore by the eosin counterstain. The proliferating mesangial cells are responsible for the inner layer of silver-positive material which can be seen in some capillary loops.

Finally, the silver staining of the basement membrane can be used to identify those conditions in which it has broken or ruptured. In the HenochSchönlein syndrome segmental necrosis of the basement membrane may be present with an overlying epithelial crescent, the formation of which is stimulated by the release of fibrin into the urinary space. Larger and more numerous crescents are associated with greater disruption of the glomerular capillary basement membranes, as seen in poly arteritis nodosa, Wegener's granulomatosis, anti-ü basement membrane antibody disease, and some-

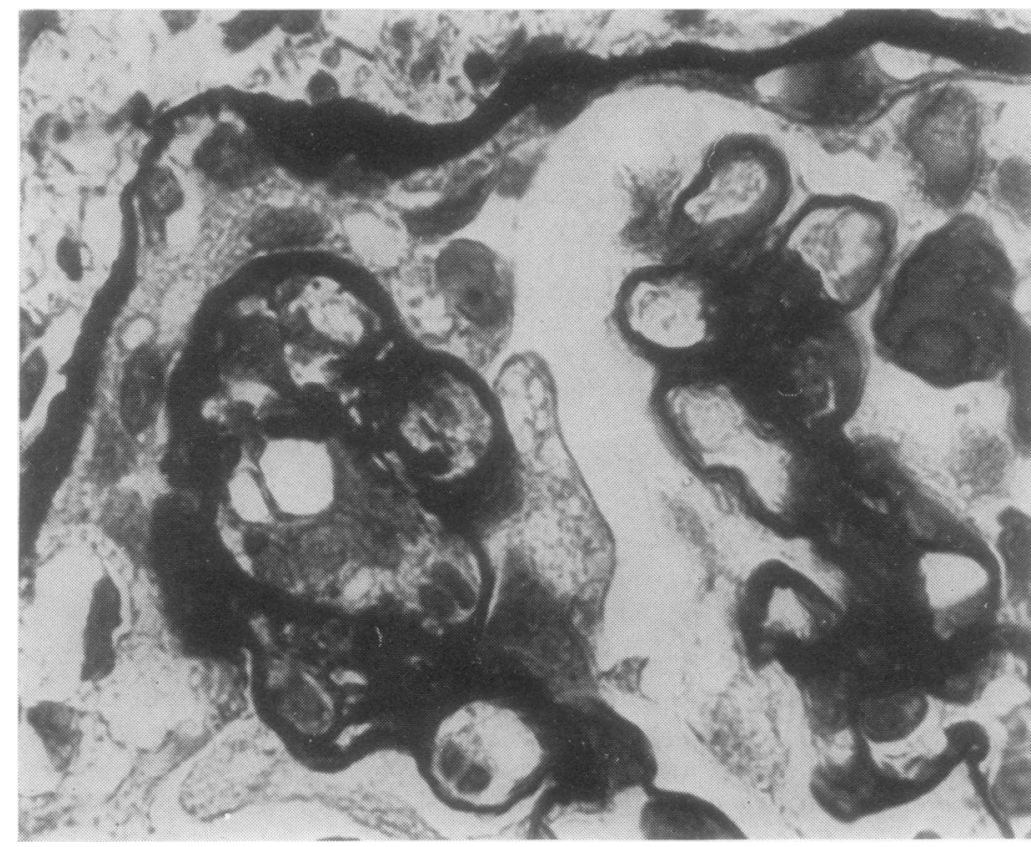

Fig. 2 A segmental sclerosing lesion from a case of focal segmental glomerulosclerosis. Jones' methenamine silver with haematoxylin and eosin counterstain $\times 1200$. 
times in severe cases of mesangiocapillary glomerulonephritis, linear dense deposit disease and even postinfectious glomerulonephritis.

TRANSMISSION ELECTRON MICROSCOPY

The gradual introduction of transmission electron microscopy occurred around the time that renal biopsies were first attempted, and although at first the detail obtained was not very precise, the technical aspects were gradually refined. This enabled a very accurate analysis of glomerular changes to be made which subsequently has been of enormous value in interpreting the appearances seen by light microscopy.

Electron microscopy of the normal glomerulus allows more precise identification of the different categories of nuclei and of their relationships to the mesangial matrix and glomerular capillary basement membranes. Ultrastructural analysis was essential for the demonstration of foot process loss which is the principal morphological abnormality seen in minimal change disease.

In membranous glomerulonephritis the electron microscope clearly differentiates between the different stages, and explains why the "spikes" can be difficult or impossible to identify by light microscopy in the early stages when they are very small. Electron microscopy was important in identifying the subepithelial "deposits" or "humps" of postinfectious glomerulonephritis with diffuse endocapillary proliferation. The hypercellularity of the glomerular tuft is accounted for in part by cellular proliferation and in part by sequestration of neutrophil polymorphs.

Electron microscopy clarifies the nature of the complex thickening of the glomerular capillary wall which occurs in mesangiocapillary glomerulonephritis. It shows the large endothelial deposits and numerous processes of mesangial cells interposed between the original basement membrane and the endothelial cell cytoplasm.

About $20 \%$ of the cases of mesangiocapillary glomerulonephritis are found to have a very different appearance by electron microscopy. Within the glomerular capillary basement membrane an electron dense material is deposited in an almost linear pattern. This is associated with mesangial cell proliferation and often with epithelial crescents. This dense deposit can also be identified in the tubular basement membranes and in Bowman's capsule. This pattern of glomerular disease has acquired the label of linear dense deposit disease, and the "dense deposit" is known to have recurred in the majority of cases which have been transplanted, although it does not usually lead to loss of the graft.

Amyloid is usually identifiable by light microscopy with the Congo red stain examined by polarised light. However electron microscopy may be valuable in identifying very small quantities of amyloid, and has explained the "spikes" formation occasionally seen by light microscopy in renal amyloidosis which may be confused with membranous change.

As mentioned earlier, basement membrane thickness is difficult to measure by light microscopy. Electron microscopy enables identification with confidence of the uniform thickening of the basement membranes which occurs in diabetic glomerulosclerosis. There are of course easier ways of diagnosing diabetes mellitus than by electron microscopy of a renal biopsy but it is sometimes necessary to consider whether, in a diabetic patient, the development of proteinuria is a complication of diabetes or due to the coincidental occurrence of some other form of glomerular disease, such as membranous glomerulonephritis.

Finally, there is one type of electron microscopic change which remains firmly in the domain of ultrastructural diagnosis and that is the basement membrane abnormality in Alport's syndrome of progressive renal failure and deafness. This inherited condition, which affects predominantly young males, presents with both proteinuria and haematuria. At this stage there may be no evidence of impaired renal function or deafness, and light microscopy of a renal biopsy may show little more than mild mesangial proliferation without immune deposits. However, electron microscopy shows a lamellation or splitting of the lamina densa, with projections from its outer layer and the inclusion of dots and granules of various sizes between the layers. Progression of the disease is associated with proliferation and sclerosis of glomeruli and tubular atrophy. The only useful light microscopical feature is the presence of interstitial foam cells which may be seen in about a third of cases. An early change in Alport's syndrome is thinning of the glomerular basement membrane, and this is the same change described for the benign familial recurrent haematuria syndrome. While it is likely that the latter represents a "forme fruste" of Alport's syndrome it is important to distinguish these two conditions for the sake of prognosis. The more widespread the typical basement membrane lamellation is found to be on electron microscopy, the greater the likelihood of Alport's syndrome. Clearly the presence of a family history, or deafness or reduced renal function makes the diagnosis relatively easy.

\section{PLASTIC EMBEDDING}

One of the valuable offshoots of electron microscopy was the discovery that thick $(1 \mu \mathrm{m})$ sections of the material embedded in plastic for electron microscopy can be stained by a variety of techniques and ex- 


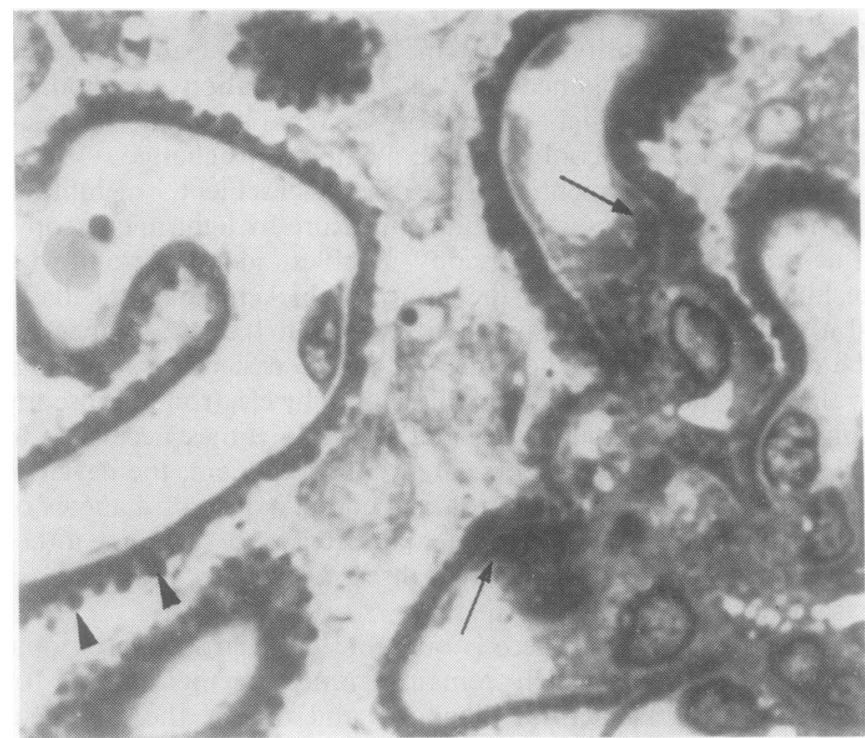

Fig. 3 Part of a glomerulus with numerous subepithelial deposits arranged along the outer aspect of the glomerular capillary basement membranes (indicated by thick arrows). This comes from a case of lupus nephritis which also shows mesangial deposits on the inner aspect of the basement membrane (thin arrows). Araldite section stained with toluidine blue $\times 1200$.

amined by light microscopy. The quality of preservation of the tissue is superior to that obtained by conventional techniques for light microscopy. Thus plastic sections provide a valuable link between light microscopy and electron microscopy.

Toluidine blue stains the linear dense deposit preferentially, and thus the diagnosis of linear dense deposit disease can be made very easily from the examination of plastic-embedded material. In lupus nephritis this method is particularly useful--for example, for illustrating the combination of membranous and mesangial proliferation (Fig. 3) or even more complex pictures with proliferation combined with subendothelial deposits.

\section{SCANNING ELECTRON MICROSCOPY}

It is perhaps unfortunate that the introduction of scanning electron microscopy should have been delayed so long, because by the time it had arrived most of the questions it could have answered had already been solved by laborious, three-dimensional reconstructions from serial electron microscopy sections. Personally I do not think that scanning electron microscopy will confer any useful advantages in the study of glomerular disease.

IMMUNOCHEMICAL TECHNIQUES

The use of immunochemical techniques in the analysis of glomerular disease has had profound effects. On the one hand they have provided markers for the identification of several reasonably distinct categories of glomerular disease which seem to be of prognostic significance. On the other hand they have had the effect of turning everyone's thoughts towards immune mechanisms although there aren many reasons for believing that non-immune mechanisms may be as important, or even more important, in the genesis of glomerulonephritis.

Initially, the problem with immunofluorescence was the need to obtain fresh tissue; this was achieved by snap-freezing part of the biopsy core, and cutting frozen sections. This inevitably meant less tissue for other techniques and also the introduction of morphological artefacts. Furthermore some antisera were non-specific.

In 1966 Graham and Karnovsky suggested the use of an alternative label to fluorescein. ${ }^{2}$ They used horse-radish peroxidase to label their antisera, whereby diaminobenzidine was oxidised to a brown end product which could be seen by conventional light microscopy. This technique could be modified for electron microscopy but still had the same basic drawbacks described for the original immunofluorescence techniques.

About two years ago we began to achieve success by adapting the immunoperoxidase technique for use on formalin-fixed paraffin-embedded material. Our first success was with tissue obtained postmortem from a patient with membranous glomerulonephritis in which IgG deposition was demonstrated (Fig. 4). After removing the paraffin wax the sections were treated for about $30 \mathrm{~min}$ with a proteolytic enzyme called Pronase. This breaks the linkage between the fixative and the proteins so that the latter can combine with peroxidase-labelled anti- 


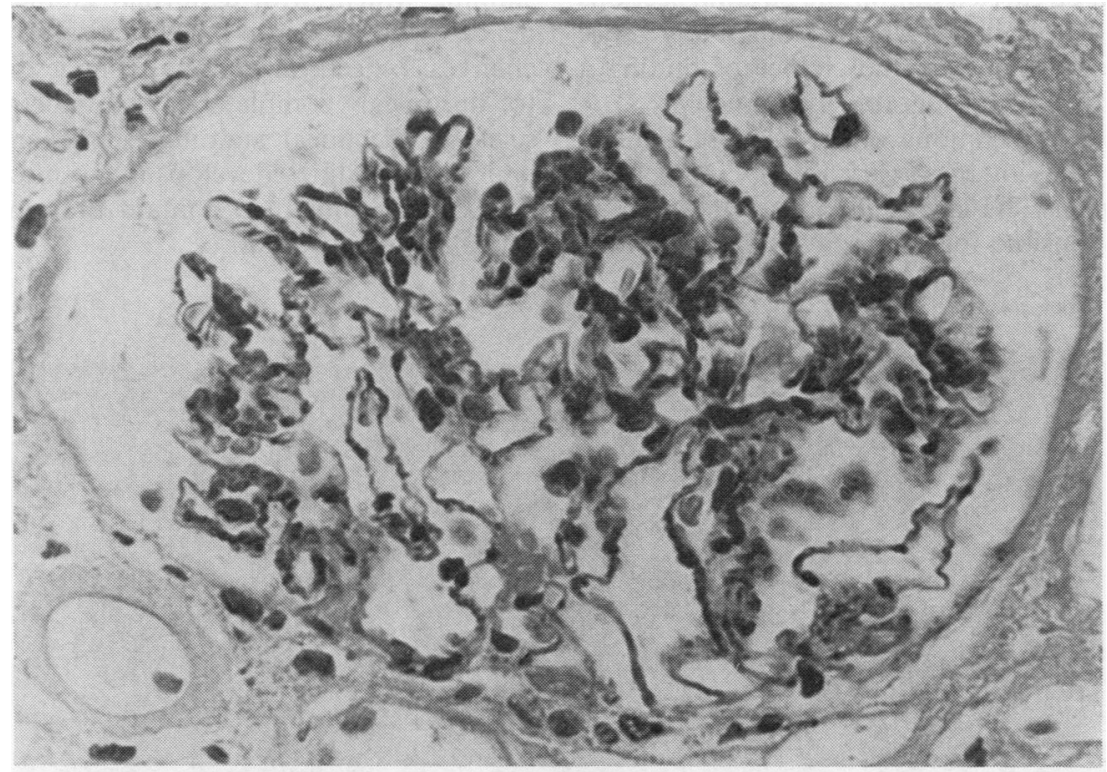

Fig. 4 Glomerulus with sparse subepithelial deposits along the capillary basement membranes from a case of membranous glomerulonephritis. Paraffin section stained with peroxidase-labelled anti-IgG $\times 320$ (cf Fig. 1).

body. The ease with which the final product can be identified is increased by adding a further peroxidaselabelled antiperoxidase antibody or PAP step to the procedure. Similarly the mesangial deposition of IgA in the recurrent haematuria syndrome can be shown by this technique. We have also found it to be of value for the identification of segmental IgM and C3 in focal segmental glomerulosclerosis (Fig. 5). The

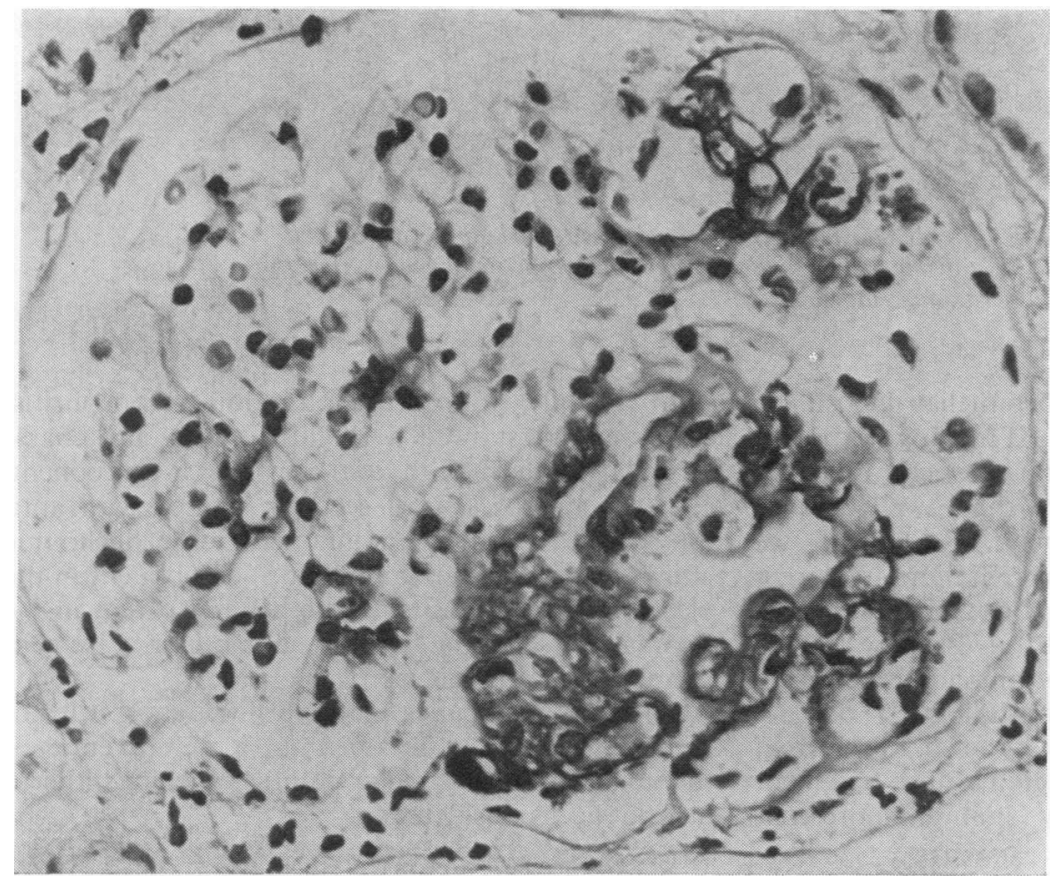

Fig. 5 Glomerulus with segmental sclerosing lesions from a case of focal segmental glomerulosclerosis. Paraffin section stained with peroxidaselabelled anti-IgM $\times 320$ (cf Fig. 2). 
fact that it can be carried out on the paraffin sections cut from the whole biopsy means that many more glomeruli are sampled and the chance of finding a segmental lesion are considerably increased.

The peroxidase technique on paraffin sections is also useful for studying mesangiocapillary glomerulonephritis (Fig. 6). The large subendothelial deposits of $\mathrm{C} 3$ are responsible for the typical pattern of this staining which, with a normal glomerulus, gives a delicate linear pattern. ${ }^{3}$ In disease this pattern changes. Indeed, in minimal change disease it tends to disappear completely. In diabetic glomerulosclerosis the linear staining is thicker than normal and when nodules develop in the mesangium these also can be shown to contain the amyloid P-component by this procedure.

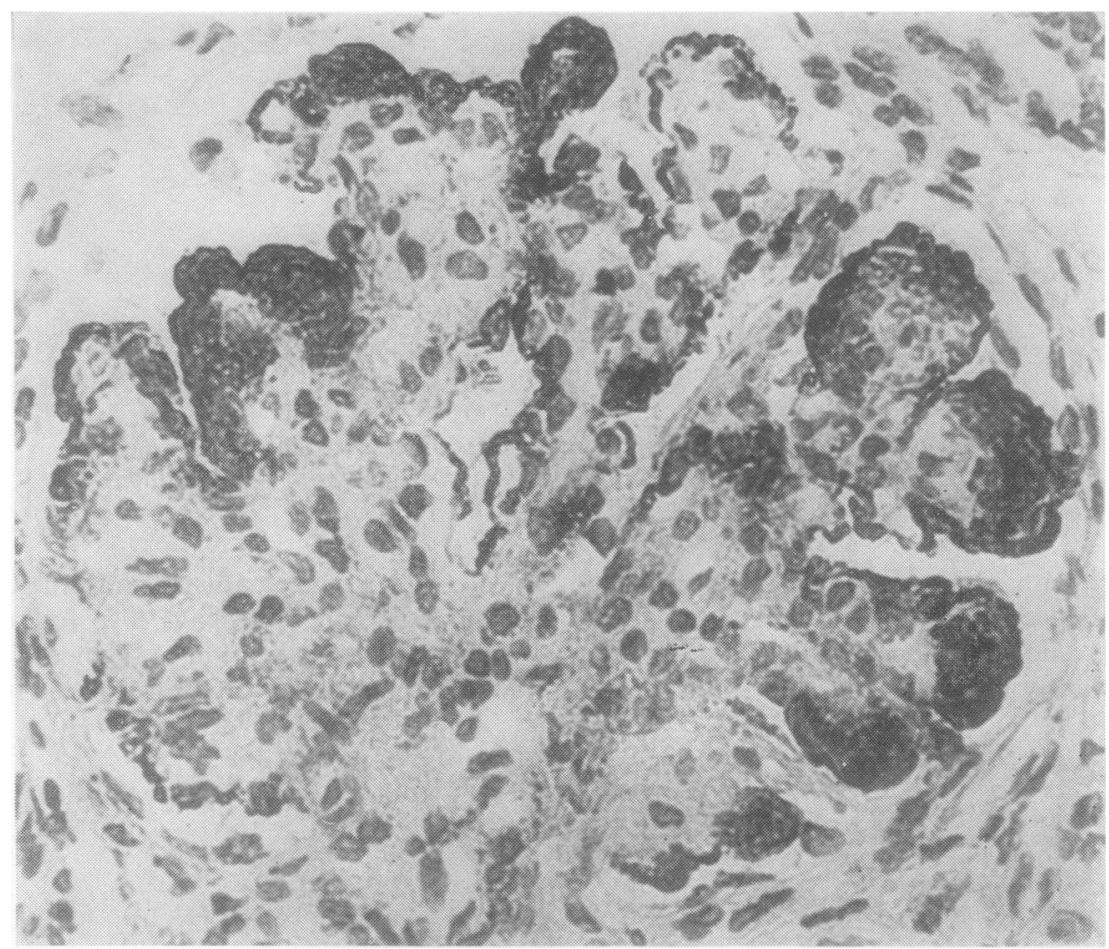

Fig. 6 Glomerulus with large subendothelial deposits and marked mesangial cell proliferation from a case of mesangiocapillary glomerulonephritis (Type I). Paraffin section stained with peroxidase-labelled anti-C3 $\times 320$.

condition. When there are subepithelial deposits as well some workers call it Type III mesangiocapillary glomerulonephritis, but so far we can find no justification for defining more than two types of mesangiocapillary glomerulonephritis. Overall we consider that the enhanced sensitivity and the larger number of glomeruli available for examination with the peroxidase method used on Pronase-treated paraffin sections are an important advance in the interpretation of glomerular morphology.

The amyloid P-component is one of the normal matrix glycoproteins of the glomerular basement membrane, as is shown by immunofluorescent
In a study of rnembranous glomerulonephritis ${ }^{3}$ all glomeruli showed a brightly fluorescing coarse granular pattern with anti-amyloid P-component. Electron microscopy of sections stained with antiamyloid $P$ by the immunoperoxidase technique showed the presence of amyloid P-component in the electron-dense deposits and to a lesser extent in the lamina rara interna of the glomerular basement membrane.

The full significance of these findings is still not yet clear but they do open up a new avenue of investigation of glomerular pathology which hitherto had not been considered. 


\section{References}

1 Jones DB. Nephrotic glomerulonephritis. Am J Pathol 1957;33:313-29.

${ }^{2}$ Graham RC, Karnovsky MJ. The early stages of absorption of injected horseradish peroxidase in the proximal tubules of mouse kidney: ultrastructural cytochemistry by a new technique. $J$ Histochem Cytochem 1966;14: 291-302.
${ }^{3}$ Dyck RF, Evans DJ, Lockwood CM, Rees AJ, Turner DR Pepys MB. Amyloid P-component in human glomerular basement membrane. Lancet 1980;ii :606-9.

Requests for reprints to: Prof. DR Turner, Department of Pathology, Musgrove Park Hospital, Taunton, Somerset. 